\title{
Link Stability with Energy Aware Ad Hoc on Demand Multipath Routing Protocol in Mobile Ad Hoc Networks
}

\author{
Senthil Murugan Tamilarasan and Kannan Eswariah \\ Department of Computer Science and Engineering, \\ Vel Tech Dr.RR and Dr.SR Technical University, Chennai, India
}

Received 2013-06-07, Revised 2013-06-27; Accepted 2013-07-10

\begin{abstract}
Mobile Ad Hoc Network is one of the wireless network in which mobile nodes are communicate with each other and have no infrastructure because no access point. The MANET protocols can be classified as proactive and reactive routing protocol. The proactive routing protocols, all nodes which participated in network have routing table. This table updated periodically and is used to find the path between source and destination. The reactive routing protocol, nodes are initiate route discovery procedure when ondemand routes. In order to find the better route in MANET, many routing protocols are designed in the recent years. But those protocols are not concentrating about communication links and battery energy. Communication links between nodes and energy of nodes are very important factor for improving quality of routing protocols. This study presents innovative Link Stability with Energy Aware (LSEA) multipath routing protocol. The key idea of the protocol is find the link quality, maximum remaining energy and lower delay. Reflections of these factors are increasing the qualities like packet delivery ratio, throughputs and reduce endto-end delay. The LSEAMRP was simulated on NS-2 and evaluation results also shown.
\end{abstract}

Keywords: AODV, AOMDV, LSEA-AOMDV, Routing Protocol, MANETs

\section{INTRODUCTION}

This Wireless Network can be classified into infrastructure wireless network and infrastructure less wireless network. In infrastructure less wireless network have three different types like Ad hoc network, Sensor Network and Mesh network. In MANETs, each node act as 'source', 'destination' and 'router'. Each node communicate with other node with the help of relay nodes as well as its used to discovering and maintaining the routes information's Zhang et al. (2011). It has some characteristics based on node mobility, changes of the network topology, battery energy, bandwidth and memory capabilities.

Chlamtac et al. (2003) notifies Mobile Ad hoc Networks (MANETs) are composed of mobile nodes, having limited resources and extreme mobility and exchanging data through wireless communication without any fixed infrastructures. Due to shorter radio transmission range, multiple hops are essentially needed for communication. Further, node mobility causes the topology of the networks to be dynamically changed, frequently re-initiating the route discovery procedure. Thus, the design of an efficient routing protocol is one of main topics in MANET and many researchers have studied this area. Recently many researchers have attended multipath routing protocol for MANETs, which can acquire multiple paths through one route discovery procedure. Multipath routing protocols provide fault tolerance for minimizing re-initiation of route discovery and load balancing for extending limited bandwidth. Additionally, load balancing makes MANETs equalizing

Corresponding Author: Senthil Murugan Tamilarasan, Department of Computer Science and Engineering,

Vel Tech Dr.RR and Dr.SR Technical University, Chennai, India 
energy consumption. When a destination node replies RREP packets to the source, intermediate nodes add their current battery status to the sum of the battery capacity field in the RREP packet in order to select data transmission route.

Applications of MANETs are battle field communication, war area, sea level communication, business environment like outside office conference, multi person decision making in game theory, vehicle communication and crisis management.

\section{ADHOC ONDEMAND DISTANCE VECTOR ROUTING (AODV)}

Li and Cuthbert (2004) declare the AODV is a single-path, on-demand routing protocol. When a source node generates a packet for a particular destination node, it broadcasts a Route Request (RREQ) packet Williams and Camp (2002). where the source and destination IP addresses remain constant for the lifetime of the network, source sequence number is a monotonically increasing indicator of packet "freshness," destination sequence number is the last known sequence number for destination node at source node and hop-count is initialized to zero and incremented at each intermediate node which processes the RREQ. Destination sequence numbers to ensure loop freedom at all times, avoiding problems associated with classical distance vector protocols Perkins et al. (2003).

Like the RREQ, a RREP is only processed on first sighting and is discarded unless it has a greater destination sequence number than the previous RREP or the same destination sequence number but a smaller hop-count. The route expiration time is the time after which the route is considered to have expired and a new route discovery process must be undertaken. Source node sends packets via the first path it hears about. If it receives a later RREP which has either fresher information or a shorter hop-count, it swaps to that, discarding the original route information. When an active route link breaks, a Route Error (RERR) packet, with sequence number incremented from the corresponding RREP and hop-count of 1, is sent by the upstream node of the broken link to source node. Upon receipt of a RERR, source node initiates a new route discovery process if it still has packets to send to destination.

\section{ADHOC ON-DEMAND MULTIPATH DISTANCE VECTOR ROUTING (AOMDV)}

Marina and Das (2002) says that Ad hoc On-demand Multipath Distance Vector (AOMDV) which is an extension of Ad hoc On-demand Distance Vector (AODV) and it's also establishes multiple loop-free and link-disjoint paths A source node floods a RREQ to the entire network in order to find routes to the destination and when the destination node receives the RREQ via different neighbors, it transmits multiple Route Reply (RREP) packets to the source node. Lee proposed a multiple routing protocol considering the residual battery capacity of route candidate nodes based on AOMDV.

When a destination node replies RREP packets to the source, intermediate nodes add their current battery status to the sum of the battery capacity field in the RREP packet in order to select data transmission route. Liu et al. (2009) introduced a threshold of the battery status of nodes. When the residual battery of intermediate nodes becomes under the threshold, they stop to flood RREQ packets and the source node switches to another route among candidates to extend network lifetime. Yoo et al. (2011) AOMDV protocol providing a route recovery mechanism when a link breaks in an active route to reduce lost packets.

\section{LINK STABILITY AND ENERGY AWARE AD HOC ON DEMAND MULTIPATH ROUTING PROTOCOL (LSEA-AOMDV)}

In the study proposed a new routing protocol which aims to acquire multiple routes having less delay between nodes in RREQ flooding phase. When a RREQ packet arrives at the destination at first, the sum of delay of intermediate nodes should be minimized and nodes with high delay nodes should avoid joining the route. The communication path will frequently breaks due to failure of path and route construction. Because of these things, increase the routing overhead and reduce the packet delivery ratio. So, controlling the routing overhead is mandatory for improve the quality of service. Routing protocols divided into two categories like topology based protocol and position based protocol. Topology based pouting is establish a path using link information. Topology based protocol, source node 
broadcast the RREQ message to neighbors and rely nodes also rebroadcasting the RREQ up to reaching the destination. The aim of the proposed routing protocol is as follows. If a node acquires the direction of the destination node then node forward the RREQ towards the direction of the destination. It is used to flooding the RREQ only towards the destination side and also avoids the flooding of route request packet away from the destination. Position based routing protocols means the node may know about position of the destination node. It is also used to flooding a RREQ towards the region of the destination only. But, the position based method is not available in many scenarios. So, we will estimate the distance or direction towards the destination. We will estimated using (a) Estimated positioning distance will be find out using the received signal strength because of node moving. (b) Estimated topological distance is used to purify the pervious method.

The Estimated Positioning Distance is used to find the quality of link between neighbor nodes. It is used to reduce the failure of the path and discover the routes again and again. AODV, AOMDV, EADB-AOMDV are on-demand routing protocol which are based on topology Tamilarasan and Eswariah (2012). In this protocol have small amount of overhead occur when they are flooding the RREQ. After finding the route, the packet will be transferred, so, minimize the rebroadcasting is main objective of this study. In proposed system, we will use global positioning system for determine the own position of the nodes. In Distance Routing Effect Algorithm for Mobility, all the nodes are update position of other nodes in routing table and also send RREQ packet towards the position of destination using position table. Link quality will be calculated from the observe of the node movement towards or away from the particular node. If the neighbor moving towards the particular node then this link may be strong or otherwise link may be weak.

\subsection{Remaining Battery Energy}

Node remaining energy can be calculated as follows:

$$
\begin{gathered}
\text { Node Remaining Energy = Node fully energy-Node } \\
\text { spent energy. }
\end{gathered}
$$

Here, node spent energy for sending and receiving the messages (RREQ, RREP and data packets).

Transmission energy can be calculated as follows:

$\operatorname{Tr}($ Energy $)=\operatorname{Tr}($ Power $) X($ Packet Size/bandwidth $)$. $\begin{aligned} & \text { here, } \operatorname{Tr}(\text { Power })= \text { Energy consumption * using voltage } \\ & \text { for transmission. }\end{aligned}$

The following formula is used to find the transmission power of particular path:

$$
\mathrm{Pj}=\sum_{i=1}^{\mathrm{n}-1} \mathrm{P}(\mathrm{Ni}, \mathrm{Ni}+1)
$$

Here:

$P j=$ Transmission power of path $\mathrm{j}$

$\mathrm{n}=$ Number of nodes participated in path $\mathrm{j}$

\subsection{Link Quality}

The link quality is obtained by:

Link Quality $=($ Transmission Radius-Distance $) /$ velocity; here, we can easily obtain distance using received signal strength of packets.

Velocity (V) between two nodes is:

$$
\mathrm{V}=\Delta \mathrm{d} / \Delta \mathrm{t}
$$

where, $\Delta \mathrm{t}$ is the time difference between the former packet receiving (time instant $\mathrm{t} 1$ ) and the next packet receiving (time instant $\mathrm{t} 2$ ) which means $\Delta \mathrm{t}=\mathrm{t} 2-\mathrm{t} 1 . \Delta \mathrm{d}$ is the distance difference between the distance $\mathrm{d} 1$ and $\mathrm{d} 2$ at, respectively, the time $\mathrm{t} 1$ and $\mathrm{t} 2$.

After finding the link quality, if the link quality is greater than threshold value of strong link, then this algorithm consider to be the path selection or otherwise it will be rejected.

\subsection{Delay}

The end-to-end delay denotes to find how long time takes for packets of data to travel across the network from source to destination. The delay represent find how long time takes for a bit of data to travel across the network from one node to another node. The proposed algorithm is to find delay between the nodes using timestamp and updating in route list. Every node update the received time of packet for to find delay like receiving time of packet in current node minus sending time of packet by previous node and also update calculated delay in routing list Cho et al. (2011).

\subsection{Lsea Algorithm}

1 if $($ node $==$ source $)$ then broadcast(RREQ) with routelist; else if(received RREP) 
then sort(routes);

using [link_Quality (strong),

Maximum(min_re_energy) and

minimum(delay)];

else rebroadcast(RREP);

endif //go to 2

2 if $($ node $==$ intermediate node $)$

calculate remaining energy

calculate delay;

update in routelist;

broadcast(RREQ) with Route_list;

endif //go to 3

3 if (node $==$ destination)

then stop broadcast(RREQ);

update as advertised hop count $=0$;

Calculate overall delay, link quality;

Update route_list,

reply (RREP) with route_list;

endif

\section{SIMULATION RESULTS}

Network Simulator is discrete event simulator and it has been used for simulating proposed algorithm with the help of network animator (nam) and gnuplot tool for performance comparisons of existing protocols like AODV, AOMDV and LSEA Yang and Wang (2011) and Gupta and Malik (2011). In simulation all nodes have the same transmission range of 500 meters and also taken times in milliseconds.

\subsection{End To End Delay}

The Fig. 1 shows the end to end delay of three protocols like AODV, AOMDV and LESA. In result comparison, LSEA perform results better than other protocols. In this simulation the values are taken every 10 milliseconds. Overall throughput increased by $22.16 \%$ because of lower end-to-end delay of the route.

\subsection{The Packet Delivery Ratio}

We observe from Fig. 2, the packet delivery ratio of LESA is substantially better than AODV and AOMDV. In fact, the packet delivery ratio for LSEA is improved by $40 \%$ over AOMDV and also the network speed considered. The explanation is the property of LESA that reduces the congestion level of the network and increases its capacity.

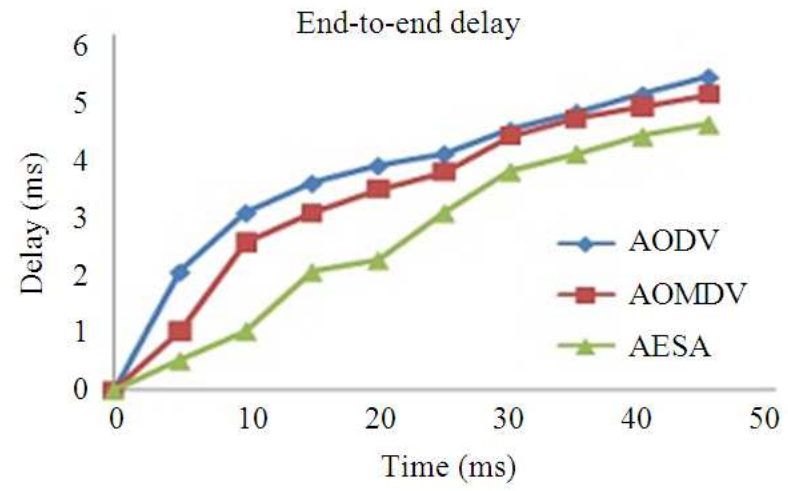

Fig. 1. End-to-end delay comparison

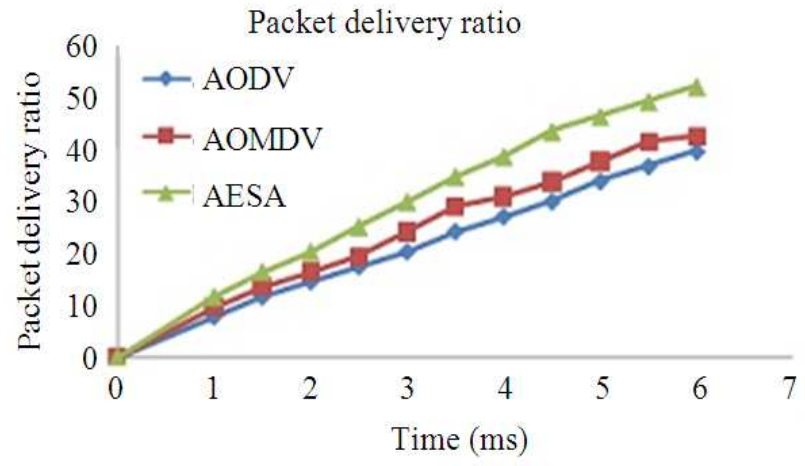

Fig. 2. Packet delivery ratio comparisons

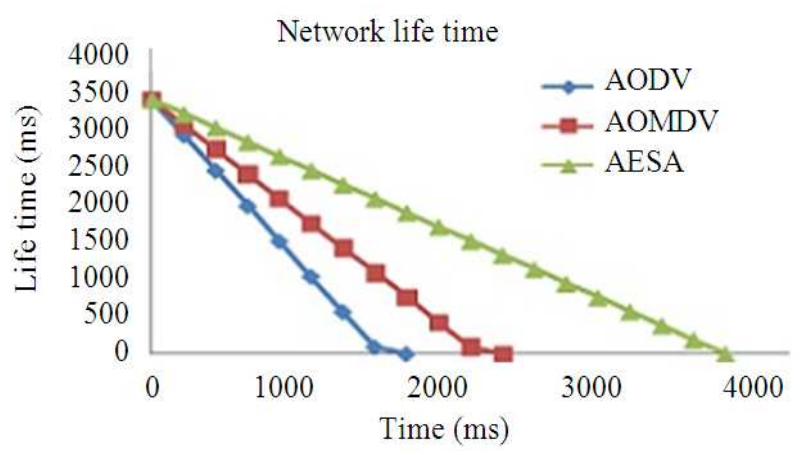

Fig. 3. Network life time comparisons

\subsection{The Life Time of Networks}

We consider the initial life time is $60 \mathrm{sec}$. Life time of network may be decreasing depends on speed of the transmissions. The speed is considered as number of packet transferred in milliseconds and it 
will be increased step by step. Figure 3 shows a lifetime of node with varying maximum node speed. Comparing with the AODV and AOMDV, the LESA Protocol increases a lifetime of a node as $23.13 \%$. The performance is enhanced as $28.58 \%$ in a circumstance that nodes have same initial delay. It shows that proposed method has been better performance in realistic circumstance.

\section{CONCLUSION}

An Link Stability and Energy Aware Ad hoc Ondemand Multipath routing Protocol (LSEA) is proposed, which adapts a delay function, link quality and battery remaining energy. It is extended from AOMDV by applying this function. Minimum and maximum delay times of this function are determined by balancing the performance improvement related to packet delivery ratio, network lifetime and side effects of time delayed flooding. Simulation results depict, LSEA improves the network lifetime in all mobility spans. In additionally, LSEA increases the packet delivery ratio because the LSEA lifetime of links becomes longer and also decreases the energy consumption. Comparing with AOMDV, the proposed algorithm is to reduce the route discovery overhead.

\section{REFERENCES}

Chlamtac, I., M. Conti, J.J.N. Liu, 2003. Mobile ad hoc networking: Imperatives and challenges. Ad Hoc Netw., 1: 13-64. DOI: 10.1016/S15708705(03)00013-1

Cho, W., D. Kim and T. Kim, 2011. Time delay ondemand multipath routing protocol in mobile adhoc networks. Proceedings of the Third International Conference on Ubiquitous and Future Networks, Jun. 15-17, IEEE XPLORE Press, Dalian, pp: 55-60. DOI: 10.1109/ICUFN.2011.5949135

Gupta, P. and A. Malik, 2011. An Ns-2 simulation based comparison of MANET routing protocols. MIT Int. J. Comput. Sci. Inform. Technol., 1: 95-101.

Li, X. and L. Cuthbert, 2004. On-demand node-disjoint multipath routing in wireless ad hoc networks. Proceedings of the 29th Annual IEEE International Conference on Local Computer Networks, Nov. 1618, IEEE Xplore Press, pp: 419-420. DOI: 10.1109/LCN.2004.90
Liu, J., Chen, J. and Y. Kuo, 2009. Multipath routing protocol for networks lifetime maximization in adhoc networks. Proceedings of the 5th International Conference on Wireless Communications, Networking and Mobile Computing, Sept. 24-26, IEEE Xplore Press, Beijing, pp: 1-4. DOI: 10.1109/WICOM.2009.5305828

Marina, M.K. and S.R. Das, 2002. Ad hoc on-demand multipath distance vector routing. ACM SIGMOBILE Mobile Comput. Commun. Rev., 6: 92-93. DOI: 10.1145/581291.581305

Perkins, C., E. Belding-Royer and S. Das, 2003. Ad hoc On-Demand Distance Vector (AODV) Routing. RFC.

Tamilarasan, S.M. and K. Eswariah, 2012. Energy aware and delay based ad hoc on-demand multipath distance vector routing in MANETs. Eur. J. Sci. Res., 85: 452-459.

Williams, B. and T. Camp, 2002. Comparison of broadcasting techniques for mobile ad hoc networks. Proceedings of the 3rd ACM International Symposium on Mobile Ad Hoc Networking and Computing, Jun. 09-11, ACM Press, Lausanne, Switzerland, pp: 194-205. DOI: $10.1145 / 513800.513825$

Yang, Y.J. and X.M. Wang, 2011. Improvement and analysis of multipath routing protocol AOMDV based on CMMBCR. Proceedings of the 7 th International Conference on Wireless Communications, Networking and Mobile Computing, Sep. 23-25, IEEE Xplore Press, Wuhan, pp: 1-4. DOI: 10.1109/wicom.2011.6040298

Yoo, D., G. Jin, B. Jang, L.T. Tuan and S. Ro, 2011. A modified AOMDV routing protocol for maritime inter-ship communication. Proceedings of the International Conference on ICT Convergence, Sept. 28-30, IEEE Xplore Press, Seoul, pp: 605-607. DOI: 10.1109/ICTC.2011.6082694

Zhang, X.M., E.B. Wang, J.J. Xia and D.K. Sung, 2011. An estimated distance-based routing protocol for mobile ad hoc networks. IEEE Trans. Veh. Technol., 60: 3473-3484. DOI: 10.1109/TVT.2011.2158865 